\title{
Estudio de las percepciones de mujeres sobre las prácticas de balance vida-trabajo en el sector bancario peruano
}

Study of women's perceptions about life-work balance practices in the sector peruvian banking

\author{
Miluska Chaparro Angulo \\ Grupo de Investigación en Gestión de la Diversidad (PUCP) \\ miluska.chaparro@pucp.pe \\ Nicole Tamayo Gutierrez \\ Grupo de Investigación en Gestión de la Diversidad (PUCP) \\ nicole.tamayo@pucp.edu.pe \\ Carolina Tantapoma Vilchez \\ Grupo de Investigación en Gestión de la Diversidad (PUCP) \\ c.tantapoma@pucp.edu.pe
}


La gestión de la diversidad de género para las empresas se basa en el beneficio que las mujeres representan para las empresas, especialmente en el sector bancario; por lo tanto, las prácticas de balance vida-trabajo representan una alternativa a favor de esta necesidad para las mujeres. El presente estudio ${ }^{1}$ tiene por objetivo conocer las percepciones de un grupo de mujeres profesionales con edades de 18 a 35 años sobre el balance vida-trabajo a través de un estudio de caso realizado en uno de los principales bancos peruano. Para ello, se analizan las relaciones estadísticas que pueden existir entre las determinantes de la mujer, la percepción de existencia y la percepción de importancia de las prácticas de balance vidatrabajo. El análisis de las determinantes concluyó en la creación de tres subgrupos, llamados perfiles. Para el análisis de percepciones, se construyó un instrumento denominado Nimica, el cual fue aplicado a 128 colaboradoras. Los principales resultados incluyen la constatación de que la percepción de importancia de cuatro prácticas se encuentra estadísticamente relacionada con la percepción de existencia y con los perfiles de las encuestadas.

Palabras clave: balance vida-trabajo, diversidad de género, gestión de estudios de mujeres, sector bancario en el Perú

Managing gender diversity for companies is based on the benefit that women represent for companies, especially in the banking sector; thus, work-life balance practices represent an alternative in favour of this requirement for women. The objective of this study is to know the perceptions of a group of professional women between 18 and 35 years of age on the life-work balance in a case study in one of the most important Peruvian banks. Therefore, the study analyzes the statistical relationships that may exist between the determinants of women, the perception of existence and the perception of importance of the life-work balance. The analysis of the determinants concluded in the creation of three subgroups, called profiles. For the analysis of perceptions, we designed a validated instrument called Nimica, which was applied to 128 collaborators. As a result, it was found that the perception of importance of four practices is statistically related to the perception of existence and the profiles of the respondents.

Keywords: work-life balance, gender diversity, management of women's studies, banking sector in Peru

1 Este trabajo tiene como base la tesis de licenciatura de las autoras (Miluska Chaparro, Nicole Tamayo y Carolina Tantapoma). 


\section{Introducción}

La globalización ha acentuado la competitividad de las empresas debido a la alta competencia a la que se encuentran expuestas en la actualidad. Las personas tienen mayor acceso a la información y, por ende, la decisión respecto a qué empresa elegir como lugar de trabajo también se vuelve más compleja. Por otro lado, actualmente se vive una guerra entre las empresas por atraer y retener el mejor talento (ManpowerGroup, 2013). Según ManpowerGroup (2017), en Perú, el 46 \% de los empleadores tiene dificultades para cubrir vacantes. Por tal motivo, las empresas han tenido que adaptar sus prácticas laborales o brindar mayores beneficios con el fin de hacer más atractivo el lugar de trabajo.

La situación se vuelve más crítica en el grupo etario de 18 a 35 años, que presenta los mayores índices de rotación laboral (Navassi, 2013); asimismo, representan la mayor fuerza laboral en el mundo. Futuro Labs (2014) afirma que a nivel mundial este grupo reúne cerca de 2300 millones de personas y en el Perú a 1700000 limeños, lo cual equivale al $35 \%$ de la población nacional total. Para este grupo etario, el balance entre la vida personal y laboral resulta ser una de las tres prioridades principales (EY, 2017); es más, quienes lo componen reconocen que son capaces de sacrificar incluso parte de su remuneración con el fin de obtener dicho balance (Insead, 2014). Como es de esperar, un segmento de la fuerza laboral de este rango etario se encuentra compuesto por mujeres.

Respecto a lo recién mencionado, se debe tener en cuenta que la participación laboral de la mujer se ha incrementado en los últimos años. En América Latina, representó el 50,2 \%, el mayor índice hasta el 2017 (OIT, 2018). Asimismo, en la empresa se reconoce el valor de la mujer a partir de su capacidad de adaptabilidad y para trabajar en entornos interdisciplinarios, entre otros atributos (León, 2004). Este rol en el mercado laboral y los roles tradicionales asociados a la mujer (cuidado del hogar, de los hijos y las personas dependientes) han generado conflictos entre su vida personal y laboral (Salgado, 2011); por lo mismo, son ellas quienes pueden verse principalmente favorecidas por las prácticas empresariales en favor del balance vida-trabajo.

Entonces, resaltando la necesidad de las empresas de ser más flexibles y brindar prácticas orientadas a los principales intereses de los jóvenes de 18 a 35 años, y siendo las mujeres quienes se encuentran en mayor desventaja en la búsqueda del balance entre su vida personal y su vida laboral, resulta de vital importancia analizar las prácticas del balance vida-trabajo a partir de las perspectivas de las mujeres.

Es así que la presente investigación tiene como objetivo general analizar la relación entre la percepción de existencia e importancia sobre las prácticas de balance vida-trabajo y cómo esta varía a partir de las determinantes de la mujer a través de un estudio de caso. Para ello, se creó un instrumento denominado Nimica. 


\section{Marco teórico}

\subsection{Mujer en el mundo laboral}

Situación actual de la mujer en el mercado laboral

La mujer tradicionalmente se vincula con el ámbito del hogar; sin embargo, poco a poco ha emergido de su esfera privada logrando participar activamente en el mercado profesional (Martínez \& Hansen, 2018). A nivel mundial, según la Organización Internacional del Trabajo (OIT) (2018), la mujer presenta una tasa de participación en el mercado laboral de 48,5 \%, un 26,5 \% más bajo que la de los hombres. Esta brecha ha ido reduciéndose en $2 \%$ desde 1990 y la mayor reducción se dio hasta el año 2009; sin embargo, en los años posteriores esta viene desacelerándose e incluso se detuvo durante los años 2011 y 2018 (OIT, 2018). Esta situación podría representar en los próximos años el retroceso de los logros obtenidos en materia de igualdad de género en el acceso al trabajo.

En América Latina y el Caribe la participación femenina en el mercado de trabajo del año 2017 superó el 50 \% por primera vez al ubicarse en 50,2 \%, lo cual significó que más de 117 millones de mujeres eran parte de la población económicamente activa (OIT, 2018). Además, la Comisión Económica para América Latina y el Caribe (CEPAL) (citado en Álvarez et al., 2016) proyecta que la participación de la mujer se incrementará en 56,4 \% para el 2020. Cabe indicar que la incorporación de la mujer latinoamericana a la población económicamente activa se ha logrado gracias al ingreso de ellas a empleos dependientes y a la creación de sus propias oportunidades de empleo (Pomar \& Martínez, 2007).

En el Perú, la población económicamente activa estuvo conformada por 7439600 mujeres (Gestión, 2017b) durante el año 2016, según datos del Instituto Nacional de Estadística e Informática (INEI). Asimismo, parece ser que el sector bancario es uno de los que presenta mayor tasa de participación femenina. También, según Asbanc (El Peruano, 2017), este sector presentó una tasa de participación femenina de 38,7 \% en el 2017, un valor mayor a la tasa de participación de este género dentro de la fuerza laboral formal a nivel nacional.

Lamentablemente, la actual crisis sanitaria ha tenido un fuerte impacto en la inclusión de la mujer en el mundo laboral a nivel de América Latina; es más, la OIT (2020) incluso indica que «existe el riesgo de que se produzca un retroceso con respecto a algunos avances logrados en los últimos decenios, y [...] que se exacerbó la desigualdad de género en el mercado laboral» (p. 2). En tiempos prepandémicos se estima que la mujer dedicaba más del triple de tiempo que los hombres al trabajo doméstico y de cuidado no remunerado (CEPAL, 2020). Aún no se tiene datos que permitan probar la hipótesis de un incremento de la carga del cuidado no remunerado en tiempos de pandemia; sin embargo, se estima un aumento significativo tomando como base el punto de partida y la cantidad de tiempo que ahora gastan las personas que teletrabajan. Al no equilibrarse la responsabilidad de los

cuidados en el hogar y un mejor balance de género en el reparto de tareas domésticas, es muy probable que esto resulte en más horas de trabajo no remunerado para las mujeres, afectando su número de horas disponibles para trabajar y su productividad (PNUD, 2020). 
La mujer se caracteriza por su capacidad de adaptación, promover el trabajo en equipo y la interdisciplinaridad. Según León (2004), en entornos cambiantes puede improvisar y realizar múltiples actividades con mayor facilidad. Además, las mujeres son poseedoras de una mayor sensibilidad para detectar las necesidades del entorno, lo que les permite negociar y resolver los problemas con mayor facilidad, resaltando su capacidad de escucha, intuición y comunicación para un trabajo en equipo más participativo y colaborativo (Chinchilla \& Jiménez, 2013). De esa manera, las cualidades particulares de la mujer promueven un mejor clima laboral en beneficio de la organización (Chinchilla, 2009).

La visión humanista de la mujer promueve que el trabajo sea compartido con otras esferas de la vida. En este sentido, la mujer puede ser un agente educador de su entorno laboral con la creación de una cultura organizacional que fomente el interés por otros aspectos de la vida. Así, contribuye a reducir la adicción al trabajo y, por el contrario, impulsa el desarrollo personal y familiar a la par del crecimiento profesional del colaborador (Conesa \& Ferrer, 2015).

La presencia de la mujer en las organizaciones representa un cambio en la forma de gestionarlas. En este sentido, urge un cambio de paradigma que considere las cualidades femeninas a la gestión cotidiana y se aproxime a la "economía del cuidado, empatía, tenacidad y afecto en las relaciones laborales, así como humanidad y calidez en los puestos de trabajo» (Chinchilla, 2013, p. 56).

Principales barreras para la inclusión de la mujer

A pesar de la inclusión de la fuerza laboral femenina y el valor que representa para las organizaciones, la mujer aún enfrenta dificultades para insertarse y desempeñarse laboralmente. En esta línea, una de las principales barreras es el reto para la mujer de lograr desempeñarse de manera exitosa laboralmente, y seguir cumpliendo con sus responsabilidades del hogar y con el cuidado de los hijos. Salgado (2011) sostiene que el equilibrio entre el trabajo y la vida familiar representa un problema para las mujeres de todo el mundo. La mujer enfrenta un conflicto en los diversos papeles que ejerce día a día como ama de casa, madre y cuidadora de personas dependientes, entre otros (Álvarez et al., 2016). En este sentido, la progresiva inserción de la mujer al mercado laboral genera impacto en los ámbitos personal, familiar y laboral, por lo que cobra importancia la búsqueda de un adecuado balance vida-trabajo que le permita cumplir con todos sus roles. Como indica la OIT (2020), estas barreras se han visto incrementadas, pues si en situaciones normales las mujeres se encargaban de tres cuartas partes del trabajo de prestación de cuidados no remunerado, durante la crisis sanitaria — con el cierre de escuelas, los niveles de enfermedad y la falta de servicios domésticos - estos niveles han incrementado de manera preocupante.

\subsection{Determinantes}

Para estudiar a las mujeres en el mercado laboral, debemos considerar variables condicionantes y demográficas, también llamadas determinantes, que permita analizarlas, 
teniendo en cuenta características de su familia y contexto (Zúñiga, 2002). Hay tres determinantes individuales planteados por Zúñiga (2002): "edad», «número de hijos» y «nivel laboral».

Adicionalmente, se incluyó "tiempo en la empresa» como un determinante necesario, pues según especialistas de recursos humanos que se entrevistaron, el tiempo mínimo para que un trabajador conozca todas las características de una empresa es de un año. Por tal motivo, el estudio cuenta con cuatro determinantes.

\subsubsection{Edad}

Es un condicionante porque según la etapa del ciclo de vida (niñez, juventud, adultez) de la mujer y el contexto en el que se encuentre, esta asumirá mayores responsabilidades (Zúñiga, 2002). Por otro lado, la investigación revela que los intereses de los grupos de edad dentro de la fuerza laboral están condicionados a los contextos de vida que experimentan. El presente estudio tiene como muestra a trabajadores de 18 a 35 años de un banco. Respecto a este rango de edad, EY (2017) señala que existen dos subgrupos con diferentes actitudes e intereses hacia el trabajo y la vida: los primeros son jóvenes de 20 a 27 años y los segundos de 28 a 35 años.

\subsubsection{Niños}

La ausencia, la presencia y el número de hijos es un factor relevante para las personas a la hora de valorar un beneficio que favorece su conciliación personal y laboral, especialmente en el caso de las mujeres, ya que existen investigaciones que argumentan que cuanto más hijos tenga una mujer, menor participación tendrá en el mercado laboral (Zúñiga, 2002). Asimismo, Ipsos (2018) revela que el 57 \% de los jóvenes peruanos en este grupo de edad (18 a 35 años) tiene hijos y el $39 \%$ asume el rol de jefe de hogar.

\subsubsection{Nivel de trabajo}

Cada nivel de la estructura de la empresa tiene sus responsabilidades y cuanto más alto es el nivel, mayores son estas. Es más complicado para las personas lograr un equilibrio personal y laboral en un nivel superior de la jerarquía, por lo que este factor se considera relevante para la presente investigación; y aún más porque el 25 \% de los jóvenes peruanos asume cargos gerenciales y supervisores en las empresas donde laboran (Gestión, 2017b).

\subsubsection{Tiempo en la empresa}

Para el presente estudio, este determinante es necesario, ya que el supuesto es que el número de años que una persona permanece en la empresa es directamente proporcional al conocimiento que tiene de sus prácticas. Se usó el periodo de un año como mínimo porque es el lapso que demora un trabajador en conocer las prácticas y beneficios que ofrece la empresa en todas las temporadas laborales. 


\subsection{Balance vida-trabajo}

Para seguir siendo competitivas en el mercado laboral, las empresas implementan políticas y prácticas para atraer y retener a quienes generan valor; en este sentido, deben conocer a sus grupos de interés y sus particularidades. En el caso de las mujeres, la interrelación de sus múltiples roles genera conflictos (trabajadora, madre, hija y ama de casa), produciendo estrés y deterioro de su calidad de vida y bienestar. La situación ideal es mantener el equilibrio; sin embargo, las mujeres enfrentan dificultades porque los diferentes roles parecen excluirse entre sí (Vera, 2017). En este sentido, las prácticas de conciliación son una alternativa que facilita el cumplimiento de los múltiples roles de la mujer en su vida. Ante esta situación, las empresas deben actuar a favor de este equilibrio.

Existen varias definiciones del equilibrio entre la vida laboral y personal. Una de ellas es la propuesta por Greenhaus y Beutell (1985), quienes definen este equilibrio como el desempeño de diferentes roles, tanto personales como familiares, el cual es crucial porque afecta la salud y el desempeño laboral de las personas. Por otro lado, Clark (2000) define el equilibrio trabajo-vida como la satisfacción y el equilibrio adecuado en la vida laboral y personal que presenta menor conflicto entre ellos. Finalmente, Salgado (2011) señala que el equilibrio trabajo-vida se da cuando todos los aspectos de la vida tienen espacio, tiempo y contextos, y forman parte del día a día de la persona. Además, argumenta que la conciliación puede ser una herramienta que facilite el empleo, la igualdad de oportunidades y la calidad de vida; por tanto, implica el estado ideal para cualquier persona. Este estudio utilizará la definición propuesta por Salgado, quien considera a la conciliación como el estado ideal en el que la persona logra cumplir con todos sus roles (hija, hermana, esposa, madre y trabajadora, entre otros) de la mejor manera posible.

\subsubsection{Beneficios de la gestión del balance vida-trabajo}

La adecuada gestión del balance vida-trabajo en las organizaciones trae consigo beneficios a los colaboradores y a la misma organización, tal como se presentará a continuación.

- Beneficios a los colaboradores: el balance vida-trabajo genera beneficios para los empleados, ya que los motiva y satisface, contribuyendo a la mejora de su autoestima. Los empleados se sienten valorados, pues tienen acceso a prácticas orientadas a su bienestar y pueden alcanzar un estilo de vida equilibrado y saludable. Además, reciben la oportunidad de desarrollar otros aspectos de su vida, mejorar su salud, y controlar su ansiedad y culpa por no atender otras responsabilidades personales (Ramírez, 2017; Red Concilia, 2007).

- Beneficios a la organización: las empresas deben promover prácticas de balance vida-trabajo pues favorecen a la creación de una ventaja competitiva. Del Castillo et al. (2011) mencionan que esta ventaja contribuye a que la organización sea considerada como un empleador atractivo e interesante para el mejor talento, creando o favoreciendo de esta manera la marca empleadora. De igual forma, favorece el compromiso del colaborador, lo cual facilita su 
retención y disminuye los costos de rotación, sustitución y reincorporación (CEPAL, 2005; Red Concilia, 2007). Finalmente, la Red Concilia (2007) resalta que el balance vida-trabajo mejora el clima laboral, factor que contribuye a la motivación y satisfacción del trabajador. En consecuencia, este equilibrio aumenta la productividad y eso se traduce en mayores ingresos para la empresa.

\subsubsection{Prácticas del balance vida-trabajo en organizaciones}

Una encuesta realizada por el Families and Work Institute (FWI) revela que un alto número de empleados cambiaría algunas compensaciones y beneficios con el objetivo de recibir medidas de flexibilidad que permitan lograr un equilibrio entre su vida personal y familiar (citado en Chinchilla et al., 2003). Parada et al. (2016) sostienen, por su parte, que las prácticas del balance vida-trabajo están sujetas a la interrelación de un contexto cultural, económico y legal, la cual se debe considerar para hacer realidad medidas organizacionales de acuerdo a los requerimientos de los trabajadores. En tal sentido, los investigadores mencionan que en Europa las prácticas del balance vida-trabajo son más promovidas debido a que se encuentran institucionalizadas tanto en las empresas como en el Gobierno, a diferencia de lo que sucede en Latinoamérica, donde, pese a reconocer su importancia, hay muchas prácticas que no se encuentran institucionalizadas.

El primer acercamiento de las prácticas del balance vida-trabajo se dio mediante la teoría. Para ello, se realizó un análisis de las cuatro principales fuentes del balance vidatrabajo: la Red Concilia (2007), Chinchilla et al. (2003), IFREI 2011 Perú (Chinchilla \& Las Heras, 2011) y el Ministerio de Trabajo y Promoción del Empleo del Perú (MTPE) (2014).

La primera fuente aborda las prácticas desde un entorno en donde se ha demostrado un mayor interés por parte de las empresas y el Estado español por implementarlas dentro de su estructura organizacional. Por otro lado, la segunda brinda las prácticas obtenidas de una encuesta realizada a 150 empresas españolas; mientras que la tercera fuente contextualiza el estudio realizado por Chinchilla et al. (2003) al ámbito laboral peruano. La cuarta fuente, por último, a diferencia de las anteriores, que abordan el tema desde una perspectiva empresarial, permite conocer la propuesta planteada por el Estado peruano con la finalidad de que las empresas puedan adoptar las prácticas de balance vidatrabajo dentro de sus organizaciones.

Lo mencionado permitió identificar, a partir de las principales fuentes, 37 prácticas de balance vida-trabajo; sin embargo, tuvieron que pasar un proceso de contextualización al sector bancario peruano. Dicho proceso será explicado en la metodología.

\subsection{Empresa $A B C$}

Las empresas del sistema bancario son aquellas "cuyo negocio principal consiste en recibir dinero del público en depósito o bajo cualquier otra modalidad contractual, y en utilizar ese dinero, su propio capital y el que obtengan de otras fuentes de financiación en conceder créditos en las diversas modalidades» (SBS, 2017, p. 43). En otras palabras, la banca busca captar recursos y otorgar créditos en diversas modalidades. 
A través de los años el sistema bancario ha mostrado un buen desempeño, de ahí su importancia para la economía del país. En relación a ello, Asbanc (2016) sostiene que la banca ha contribuido con el bienestar y el dinamismo de la economía nacional aún en tiempos de crisis políticas y económicas.

Por otro lado, un aspecto importante es el interés de los jóvenes peruanos en trabajar en este sector. En esta línea, E. R. Ronald (Gestión, 2013) señala que el 27 \% de ellos tiene el interés en desempeñarse en consultorías, el $26 \%$ busca realizarse en la banca y el $21 \%$ en el sector retail. Por lo mencionado, el presente estudio sitúa la investigación en el sector bancario; y, además, se eligió a la empresa ABC debido a su importancia en el sector bancario. Dentro de los factores de importancia se encuentra su participación en el grupo Aequales (2019), el cual busca reducir las brechas laborales entre hombres y mujeres. Asimismo, se consideró su reconocimiento en el ranking Great Place to Work (Empresa ABC, 2018) para mujeres por su buena gestión.

\section{Metodología}

La estrategia general de la investigación es el método de estudio de casos, pues se busca la comprensión de un fenómeno bajo su propio contexto, tal como lo menciona Harrison (citado en Ponce \& Pasco, 2015). En principio, se decidió desarrollar un caso de estudio único en el sector bancario peruano. Como se mencionó, se trabajó con uno de los cinco bancos más importantes del país, reconocido por entidades como Aequales (2019) y Great Place to Work (Empresa ABC, 2018) como un buen lugar de trabajo para mujeres.

\subsection{Muestra}

Dentro del caso de estudio, la investigación se centró en una muestra de mujeres de 18 a 35 años debido a que la literatura sostiene que la población perteneciente a dicho rango etario presenta un mayor interés por conciliar su vida laboral y personal. Asimismo, se eligió a mujeres de la sede central del banco, puesto que representan el mayor porcentaje de mujeres en ese rango etario de todas las sedes de la compañía (40 \%); además, según la jefa de Experiencia del Colaborador (comunicación personal, 19 de octubre de 2018), la sede central es la que presenta mayor acceso a las prácticas de balance vida-trabajo ofrecidas.

De esta manera, el perfil de las encuestadas presenta las siguientes características a partir de las determinantes teóricas:

- Rango etario: mujeres de 18 a 35 años.

- Antigüedad en la empresa: mínimo un año.

- Cargo administrativo: gerente, subgerente, jefa, supervisora, analista/ ejecutiva y asistente.

- $\quad$ Sede de trabajo: sede central en Lima, ABC.

Por último, tomando en consideración las condiciones y accesibilidad al banco, se optó por el muestreo no probabilístico por conveniencia, técnica que permite tomar 
el mayor número de casos accesibles (Otzen \& Manterola, 2017). Es así que, de las 310 colaboradoras que cumplían con el perfil, se obtuvo 128 encuestas efectivas.

\subsection{Construcción del instrumento Nimica}

Para recoger y medir las variables, fue necesaria la construcción de un instrumento al que se denominó Nimica, lo cual se explicará más adelante.

\subsubsection{Identificación de prácticas del balance vida-trabajo en el sector bancario peruano}

Como se mencionó anteriormente, las prácticas de balance vida-trabajo están directamente relacionadas con el contexto y es por ello que esta investigación propuso la construcción de un instrumento —al que se denominó Nimica - para recoger y medir las variables necesarias. La identificación de las prácticas del balance vida-trabajo desde la teoría hasta el contexto bancario peruano consistió en cuatro pasos.

El primer paso fue la revisión teórica de las cuatro principales fuentes del balance vida-trabajo: la Red Concilia (2007), Chinchilla et al. (2003), IFREI 2011 Perú (Chinchilla \& Las Heras, 2011) y el MTPE (2014). Como resultado, se logró un primer acercamiento desde la literatura al balance vida-trabajo.

Luego, el segundo paso consistió en identificar las prácticas comunes y no comunes de las fuentes con el objetivo de organizar la información y obtener un consenso de la literatura revisada. Así, se obtuvo una adaptación propia de la denominación y definición de 37 prácticas luego de homogeneizarlas y dividirlas por sus definiciones.

El tercer paso consistió en entrevistar a tres expertos académicos peruanos en conciliación vida-trabajo para validar la existencia y definición de la lista de prácticas del paso previo. La trayectoria en el ámbito académico y las investigaciones sobre el tema balance vida-trabajo en el contexto peruano fueron los criterios para elegirlos. A través de entrevistas a profundidad, solicitamos a los expertos analizar y validar las prácticas para la realidad peruana, indicando si la práctica era relevante o no por medio de cuatro posibles respuestas (sí, no, probablemente sí, probablemente no). Luego, medimos el I-CVI (índice de validez de contenido para elementos) y consideramos las prácticas con un índice de 0,5 o más (Bendaravičienè, 2014). Este consenso resultó en una lista de 28 prácticas laborales adaptadas al contexto peruano.

En el cuarto paso, se realizaron entrevistas exhaustivas a siete especialistas en recursos humanos y de recursos humanos en el sector bancario para corroborar las 28 prácticas laborales identificadas y contextualizarlas al sector bancario peruano mediante los mismos análisis, la aplicación del I-CVI y la validación de prácticas con índice de 0,5 o más. El resultado final son diez prácticas de conciliación contextualizadas al sector bancario peruano y presentadas en la revisión de la literatura. Estas prácticas fueron agrupadas en seis categorías: flexibilidad en el tiempo, apoyo familiar, información, apoyo profesional, beneficios corporativos y extrasociales, y salud (ver tabla 1). 
Tabla 1. Lista de prácticas de contextualizadas al sector bancario peruano

Listado de prácticas balance vida-trabajo para el instrumento Nimica

\begin{tabular}{|c|c|c|c|}
\hline$\#$ & Categoría & Denominación de la práctica & Definición \\
\hline 1 & $\begin{array}{l}\text { Flexibilidad en el } \\
\text { tiempo }\end{array}$ & $\begin{array}{l}\text { Horario flexible de trabajo por } \\
\text { resultados }\end{array}$ & $\begin{array}{l}\text { Horario flexible de trabajo por resultados } \\
\text { (no es necesario cumplir las ocho horas } \\
\text { laborales por día) }\end{array}$ \\
\hline 2 & & $\begin{array}{l}\text { Horario flexible por motivo de } \\
\text { educación superior }\end{array}$ & $\begin{array}{l}\text { Flexibilidad de horarios para realizar } \\
\text { estudios de educación superior }\end{array}$ \\
\hline 3 & \multirow[t]{2}{*}{ Apoyo familiar } & Permisos excepcionales & $\begin{array}{l}\text { Permisos médicos a los trabajadores } \\
\text { para acompañar la atención en salud de } \\
\text { sus hijos u otros dependientes, o para } \\
\text { presentaciones escolares, cumpleaños, } \\
\text { etc. (puede ser entregado bajo la } \\
\text { modalidad de cuponeras anuales) }\end{array}$ \\
\hline 4 & & $\begin{array}{l}\text { Actividades recreativas para hijos } \\
\text { en vacaciones escolares }\end{array}$ & $\begin{array}{l}\text { Actividades recreativas o de formación } \\
\text { en las épocas de vacaciones escolares } \\
\text { para los hijos de los trabajadores }\end{array}$ \\
\hline 5 & \multirow[t]{2}{*}{ Información } & Asesoramiento personal & $\begin{array}{l}\text { Talleres y seminarios de desarrollo de } \\
\text { habilidades blandas y asesoramiento } \\
\text { para solucionar problemas individuales } \\
\text { (personales, financieros, familia, estrés, } \\
\text { matrimonio, etc.) }\end{array}$ \\
\hline 6 & & $\begin{array}{l}\text { Seminarios, talleres o sesiones } \\
\text { de información sobre la concilia- } \\
\text { ción de la vida laboral y familiar }\end{array}$ & $\begin{array}{l}\text { Seminarios, talleres o sesiones de } \\
\text { información sobre la conciliación de la } \\
\text { vida y el trabajo, por ejemplo: cursos de } \\
\text { padres y educación de los hijos, manejo } \\
\text { de conflictos trabajo-familia, gestión } \\
\text { del tiempo, gestión del estrés, gestión } \\
\text { conflictos }\end{array}$ \\
\hline 7 & Apoyo profesional & Asesoramiento profesional & $\begin{array}{l}\text { Mentoría profesional para desarrollar } \\
\text { el potencial de ejecutivos en términos } \\
\text { organizativos y laborales }\end{array}$ \\
\hline 8 & $\begin{array}{l}\text { Beneficios } \\
\text { corporativos y } \\
\text { extrasociales }\end{array}$ & Beneficios corporativos & $\begin{array}{l}\text { Beneficios corporativos en subvención } \\
\text { o descuento para cursos de extensión o } \\
\text { educación superior, alimentos, club social, } \\
\text { biblioteca, premios de antigüedad, playa } \\
\text { de estacionamiento gratuita, gimnasio, } \\
\text { viajes, ropa, taxi, tasas preferenciales para } \\
\text { préstamos y deporte } \\
{ }^{*} \text { Algunos beneficios en específico pueden } \\
\text { ir cambiando de acuerdo a la entidad }\end{array}$ \\
\hline 9 & \multirow[t]{2}{*}{ Salud } & $\begin{array}{l}\text { Cobertura de la EPS por parte de } \\
\text { la empresa }\end{array}$ & $\begin{array}{l}\text { La empresa ofrece cobertura parcial o } \\
\text { total del EPS en favor del trabajador }\end{array}$ \\
\hline 10 & & $\begin{array}{l}\text { Afiliación anticipada del seguro } \\
\text { vida para el trabajador }\end{array}$ & $\begin{array}{l}\text { Afiliación anticipada del seguro vida para } \\
\text { el trabajador antes de cumplir cuatro } \\
\text { años laborando en la misma empresa } \\
\text { * Según el DL 688, al cumplir cuatro } \\
\text { años en la misma empresa el empleador } \\
\text { debe asegurar al trabajador con un } \\
\text { seguro vida que cubre hasta ocho o } \\
16 \text { veces su sueldo en caso muera de } \\
\text { manera natural o en caso de accidente, } \\
\text { respectivamente }\end{array}$ \\
\hline
\end{tabular}

Fuente: adaptado de Red Concilia (2007), Chinchilla et al. (2003), IFREl 2011 Perú (Chinchilla \& Las Heras, 2011) y MTPE (2014). Asimismo, de comunicaciones personales con Pacheco (2018), Avolio (2018), Vera (2018), Beingolea (2018), Heinman (2018), Tuesta (2018), Amaya (2018), Segura (2018), Maldonado (2018) y Ramos (2018). 
Este listado de prácticas fue el insumo principal para la construcción del instrumento Nimica. Asimismo, esta lista puede ser utilizada para futuras investigaciones en entidades bancarias peruanas.

Adicionalmente, con este listado de prácticas contextualizadas al sector bancario peruano, se procedió a preguntarle al área de Recursos Humanos de ABC si efectivamente realizaban las diez prácticas en cuestión. Esto se realizó con el fin de conocer si existe una brecha entre las prácticas que el banco menciona que realiza y las que las colaboradoras perciben.

\subsubsection{Diseño y validación del instrumento Nimica}

El instrumento Nimica es una encuesta que permite recoger y medir la percepción de la colaboradora sobre la existencia e importancia de las prácticas de balance vida-trabajo en el sector bancario peruano.

Para la comprensión de dicho instrumento es necesario explicar las variables que contempla. Por un lado, se encuentran las variables de percepción (percepción de importancia y existencia de las prácticas); y, por otro, las variables consideradas como mandos de control o determinantes (edad, número de hijos, tiempo trabajando en la empresa y nivel de puesto). Cabe resaltar que ambos tipos de variables son categóricas.

Respecto a la variable categórica "percepción de existencia», se usaron respuestas dicotómicas («sí» y «no»). Cabe resaltar que se consideró importante utilizar esta variable con el fin de confirmar si la práctica que la empresa $A B C$ menciona que realiza efectivamente es percibida por las colaboradoras.

Por el lado de la variable "percepción de importancia», inicialmente se propuso aplicar la escala de Likert del 1 al 7, pues se encontró que es la escala que se usa en estudios de percepción; sin embargo, al aplicar las pruebas pilotos se encontró que a las personas no les era sencillo comprenderla y, por lo tanto, se optó por reducir la escala de Likert del 1 al 5, donde 1 equivale a muy poco y 5 a mucho.

La validación del instrumento Nimica se realizó por juicio de expertos y mediante análisis de fiabilidad al presentar un coeficiente alfa de Cronbach de 0,086, mayor al mínimo de 0,7 que comentan los autores para que una herramienta sea fiable (García-Alcaraz et al., 2015; Ketkar et al., citados en Maese et al., 2016).

\subsection{Análisis de herramientas estadísticas}

El estudio realizó un análisis correlacional, ya que se busca conocer la relación o grado de asociación entre dos o más variables en cierto contexto (Hernández et al., 2014).

Primero, para evaluar si existía interacción entre las determinantes de la mujer, se aplicó la prueba paramétrica de la chi al cuadrado. Al tener como resultado que son interdependientes, se optó por trabajarlas de manera conjunta aplicando un análisis de clúster y el análisis de regresión logística. El análisis de clúster jerárquico, por su parte, tiene como finalidad identificar y clasificar grupos, priorizando la homogeneidad dentro de cada uno y la heterogeneidad entre los grupos (Luna-Arocas \& Li-Ping, 2005). A estos subgrupos se les denominó «perfiles». 
Luego de obtener los subgrupos, se aplicó la regresión logística, un tipo de análisis en el que, a partir de un modelo estadístico, se conoce si existe una asociación entre una variable dependiente $(Y)$ y una o varias variables independientes cualitativas $(X)$ (Sanchez, citado en Fraj, 2003). Para ello, se construyeron tres modelos base de regresión logística para evaluar las relaciones.

El primer modelo se generó para corroborar qué determinantes se ajustaban al subgrupo propuesto mediante la técnica «introducir todas las variables obligatoriamente». Según Moral (2006), es un proceso de selección manual que contempla todas las variables a priori, pues se identifica y elimina la menos significativa. Este proceso se repite hasta obtener un modelo que contemple solo variables significativas. Por otro lado, debido a que las variables dependientes (perfil e importancia) pueden tomar más de dos valores, se construyeron modelos de regresión multinomial. Asimismo, ya que la variable de existencia es dicotómica, se construyó un modelo de regresión binomial.

Tabla 2. Modelos iniciales de regresión logística propuestos para el estudio

\section{Modelos de regresión logística}

\begin{tabular}{|c|c|c|c|c|}
\hline & Variables & Tipo de regresión & Finalidad & Modelo de regresión \\
\hline \multirow[t]{2}{*}{1} & (Y): perfil & \multirow[t]{2}{*}{ Multinomial } & \multirow{2}{*}{$\begin{array}{l}\text { Construcción de } \\
\text { los perfiles; es } \\
\text { decir, definición de } \\
\text { qué determinantes } \\
\text { caracterizan el perfil de } \\
\text { la colaboradora }\end{array}$} & \multirow{2}{*}{$\begin{array}{l}\text { Y (perfil) }=\boldsymbol{\beta} 0+\boldsymbol{\beta} 1 \text { (edad) }+ \\
\boldsymbol{\beta} \text { 2(tiempo laborando) }+ \\
\boldsymbol{\beta} \text { 3(número de hijos) }+ \\
\boldsymbol{\beta} \text { 4(nivel de puesto) }+\end{array}$} \\
\hline & $\begin{array}{l}\text { (X): edad, número de } \\
\text { hijos, antigüedad en } \\
\text { la empresa y nivel de } \\
\text { puesto }\end{array}$ & & & \\
\hline \multirow[t]{2}{*}{2} & $(Y)$ : existencia & \multirow[t]{2}{*}{ Binomial } & \multirow{2}{*}{$\begin{array}{l}\text { Conocer si el perfil de } \\
\text { la colaboradora afecta la } \\
\text { percepción de existencia }\end{array}$} & \multirow{2}{*}{$\begin{array}{l}Y(\text { existencia })=\boldsymbol{\beta} 0+\boldsymbol{\beta} 1 \\
(\text { perfil })+\boldsymbol{\varepsilon}\end{array}$} \\
\hline & $(X)$ : perfil & & & \\
\hline \multirow[t]{2}{*}{3} & (Y): importancia & \multirow[t]{2}{*}{ Multinomial } & \multirow{2}{*}{$\begin{array}{l}\text { Conocer si existe el } \\
\text { perfil y si la percepción } \\
\text { de existencia afecta } \\
\text { la percepción de } \\
\text { importancia }\end{array}$} & \multirow{2}{*}{$\begin{array}{l}Y(\text { importancia })=\boldsymbol{\beta} 0+\boldsymbol{\beta} 1 \\
(\text { perfil })+\boldsymbol{\beta} 2 \text { (existencia) }+\boldsymbol{\varepsilon}\end{array}$} \\
\hline & (X): perfil y existencia & & & \\
\hline
\end{tabular}

Fuente: elaboración propia.

Para determinar si la relación entre la variable dependiente e independiente es estadísticamente significativa, se aplicó la prueba ómnibus en regresiones binomiales y la prueba de verosimilitud en el caso de la regresión multinomial.

Asimismo, para cada tipo de modelo de regresión logística (binomial y multinomial) utilizamos pruebas para la bondad de ajuste con el objetivo de comprobar que el modelo propuesto puede ser explicado estadísticamente, así como otras para profundizar en la relación entre las variables, según lo propuesto por Field (2013), como R-cuadrado de Nagelkerke (R2), Hosmer-Lemeshow y Pearson. Cabe resaltar que se utilizó un nivel de confianza del $90 \%$ para todas las pruebas mencionadas. 


\section{Resultados}

A pesar de que la investigación presenta un análisis correlacional, es importante mencionar dos hallazgos descriptivos importantes: la práctica más importante para las colaboradoras de la empresa $A B C$ fue "pago de cobertura EPS» y la menos importante "actividades recreativas vacacionales para hijos».

En cuanto al análisis correlacional, el primer objetivo fue estudiar las determinantes. Se inició el proceso realizando la prueba no paramétrica de chi al cuadrado entre las determinantes, encontrando interdependencia entre las variables (tabla 3). Dicha prueba evidenció la necesidad de utilizar clústeres para agrupar variables entre sí.

Tabla 3. Chi al cuadrado entre las determinantes

\begin{tabular}{l|c|c|c|c} 
Determinantes & Edad & $\begin{array}{c}\text { Número de } \\
\text { hijos }\end{array}$ & $\begin{array}{c}\text { Antigüedad en } \\
\text { la empresa }\end{array}$ & $\begin{array}{c}\text { Nivel de } \\
\text { puesto }\end{array}$ \\
\hline Edad & - & 0,162 & 0,171 & 0,026 \\
\hline Número & - & - & 0,011 & 0,01 \\
\hline Tiempo laborando & - & - & - & 0,03 \\
\hline Nivel de puesto & - & - & - & Fuente: elaboración propia.
\end{tabular}

Frente a esa necesidad, se aplicó el análisis de clústeres jerárquicos con el método de agrupación Ward, en el que se consideró la medida de "distancia euclídea al cuadrado". Como resultado, se obtuvo un dendograma que mostró la existencia de tres subgrupos, los cuales fueron denominados «perfiles».

Para definir los «perfiles» se propone un modelo inicial de regresión logística compuesto por una variable dependiente (perfil de la encuestada) y un conjunto de variables independientes (edad, número de hijos, nivel laboral, antigüedad en la empresa).

$Y($ perfil $)=\boldsymbol{\beta} 0+\boldsymbol{\beta} 1$ (edad) $+\boldsymbol{\beta} 2$ (tiempo laborando) $+\boldsymbol{\beta} 3$ (número de hijos) + $\boldsymbol{\beta} 4$ (puesto de trabajo) $+\boldsymbol{\varepsilon}$

Este modelo de regresión logística «perfil» fue validado con la prueba de razón de verosimilitud para conocer la significancia de cada determinante. Con dicha prueba se encontró que las variables «número de hijos» y «tiempo en el trabajo» no eran estadísticamente significativas; por lo tanto, se eliminaron. Es así como quedó compuesto el modelo propuesto para la variable dependiente "perfil», solo por dos de las cuatro variables iniciales. Es decir, el perfil se encuentra explicado a partir de las variables «edad» $\mathrm{y}$ «puesto de trabajo».

Tal como se mencionó, se determinaron tres perfiles: "mujeres promesas», «mujeres seniors» y «mujeres directivas», las cuales tienen las siguientes características: 
1. Perfil «mujeres promesas»: representan el $22 \%$ de las mujeres encuestadas. Son mujeres jóvenes de 22 a 26 años, principalmente analistas (93 \%).

2. Perfil «mujeres seniors»: representa el $49 \%$ de las encuestadas. Son mujeres de 27 a 32 años, la mayoría analistas (60\%).

3. Perfil «mujeres directivas»: representa el $29 \%$ de las encuestadas. Son mujeres de 32 a 35 años y ocupan mayoritariamente cargos directivos (32\%).

Luego de conocer los perfiles, se buscó definir si estos condicionan la percepción de existencia de las encuestadas. Para ello, se aplicaron pruebas de ajustes de bondad, detectando que la percepción de dos prácticas está condicionada por el perfil de la colaboradora. Esto se evidenció a partir de la significancia en la prueba ómnibus. Así, se encontró que la percepción de la existencia de las prácticas "permisos excepcionales» y "talleres de habilidades blandas» se ve afectada por el perfil del encuestado.

El segundo objetivo fue conocer si los perfiles explicaban la existencia de las prácticas, para lo cual se aplicó el coeficiente de R2 Nagelkerke. Con esta prueba se evidenció que las variables dependientes están siendo explicadas por las independientes en un $17,1 \%$ y $6,8 \%$, respectivamente. Esto indica que puede haber otras variables independientes no consideradas en el modelo que podrían ser estudiadas más adelante.

Tabla 4. Pruebas de ajuste de bondad y clasificación porcentual: percepción de existencia

\begin{tabular}{|c|c|c|c|c|c|}
\hline \multirow{2}{*}{$\begin{array}{l}\text { Dependiente } \\
\text { (prácticas) }\end{array}$} & \multirow{2}{*}{$\begin{array}{c}\text { Variable } \\
\text { independiente }\end{array}$} & \multicolumn{3}{|c|}{ Bondad de ajuste } & \multirow{2}{*}{$\begin{array}{c}\text { Porcentaje } \\
\text { de casos } \\
\text { correctamente } \\
\text { clasificados }\end{array}$} \\
\hline & & $\begin{array}{c}\text { Prueba } \\
\text { ómnibus } \\
\text { (sig.) }\end{array}$ & $\begin{array}{c}\text { R2 } \\
\text { Nagelkerke }\end{array}$ & $\begin{array}{c}\text { Hosmer- } \\
\text { Lemeshow }\end{array}$ & \\
\hline Permisos especiales & Perfil & 0,011 & $17,1 \%$ & 1000 & $93,0 \%$ \\
\hline $\begin{array}{l}\text { Talleres de habilidades } \\
\text { blandas }\end{array}$ & Perfil & 0,037 & $6,8 \%$ & 1000 & $63,3 \%$ \\
\hline
\end{tabular}

Fuente: elaboración propia.

El tercer objetivo fue conocer si los perfiles y la percepción de existencia condicionan la percepción de importancia. En este caso, se concluyó que las respuestas de percepción de importancia, principalmente, se encontraban en las valoraciones más altas (3, 4 y 5); es decir, había sesgo hacia arriba. Por ello, se agruparon las respuestas en tres subgrupos: los valores 1, 2 y 3 se denominaron "de bajo valor», 4 como "de valor» y 5 como «muy valorado».

A partir de las pruebas de bondad, de verosimilitud y Pearson se constató que el perfil de la mujer encuestada condiciona la percepción de importancia de las prácticas "actividades recreativas para niños», "talleres de conciliación vida-trabajo» y "temprana afiliación al seguro». Asimismo, la percepción de importancia de las prácticas "afiliación anticipada de seguro de vida» y "beneficios corporativos» se encuentra condicionada por la percepción de existencia de la encuestada (tabla 5). 
Tabla 5. Pruebas de ajuste de bondad y clasificación porcentual: percepción de importancia

\begin{tabular}{|c|c|c|c|c|c|c|}
\hline \multirow{2}{*}{$\begin{array}{l}\text { Dependiente } \\
\text { (prácticas) }\end{array}$} & \multirow{2}{*}{$\begin{array}{c}\text { Variable } \\
\text { independiente }\end{array}$} & \multicolumn{2}{|c|}{$\begin{array}{c}\text { Prueba de } \\
\text { verosimilitud }\end{array}$} & \multicolumn{2}{|c|}{ Bondad de ajuste } & \multirow{2}{*}{$\begin{array}{l}\text { Porcentaje } \\
\text { de casos } \\
\text { correctamente } \\
\text { clasificados }\end{array}$} \\
\hline & & Perfil (sig) & $\begin{array}{c}\text { Existencia } \\
\text { (sig.) }\end{array}$ & Pearson & $\begin{array}{c}\text { R2 de } \\
\text { Nagelkerke }\end{array}$ & \\
\hline $\begin{array}{l}\text { Actividades } \\
\text { recreativas para } \\
\text { niños }\end{array}$ & Perfil & 0,002 & 0,239 & 0,693 & $16,50 \%$ & $52,30 \%$ \\
\hline $\begin{array}{l}\text { Talleres de balance } \\
\text { vida-trabajo }\end{array}$ & Perfil & 0,040 & 0,719 & 0,107 & $9,50 \%$ & $51,60 \%$ \\
\hline $\begin{array}{l}\text { Beneficios } \\
\text { corporativos }\end{array}$ & Perfil & 0,903 & 0,002 & 0,334 & $14,30 \%$ & $75,80 \%$ \\
\hline $\begin{array}{l}\text { Afiliación anticipada } \\
\text { de seguro de vida }\end{array}$ & Perfil & 0,016 & 0,080 & 0,232 & $9,50 \%$ & $71,90 \%$ \\
\hline
\end{tabular}

Fuente: elaboración propia.

Por otro lado, respecto al test de Nagelkerke, la percepción de importancia se explica por la percepción de existencia y el perfil en 16,5\%, 9,5\%, 14,30 \% y 9,5\% en las prácticas "actividades recreativas de vacaciones para niños", "beneficios corporativos", "talleres de conciliación» y "afiliación anticipada a un seguro de vida», respectivamente. A su vez, este resultado indica que pueden existir otras variables independientes no consideradas en el modelo que podrían ser de interés para estudios posteriores.

\section{Discusión}

La presente investigación tuvo limitaciones para su desarrollo. En primer lugar, se encontraron escasas investigaciones académicas sobre el tema y 1) la mayoría de las publicaciones son artículos que se limitan a mencionar iniciativas específicas en las empresas; 2) las investigaciones sobre la aplicación de prácticas de balance vida-trabajo destacan principalmente la importancia del tema, pero no explican su aplicación o efectividad a partir de un estudio de caso; 3) pocas fuentes aplican una metodología de investigación; $y$, finalmente, 4) las investigaciones sobre balance vida-trabajo se basan en la perspectiva de la empresa, más no en la de los colaboradores, como sí hace este estudio. Esto último dificultó la posibilidad de encontrar una metodología adecuada para esta investigación y motivó a la construcción del instrumento Nimica.

En segundo lugar, la respuesta negativa de otros bancos peruanos líderes a participar en la investigación fue otra de las limitaciones del estudio. Por esta razón, se realizó un estudio de caso único y las conclusiones no pueden ser generalizadas. Cabe mencionar que, en algunos casos, la negativa se debió a la ausencia de prácticas de balance vida-trabajo institucionalizadas en la organización. Asimismo, por cuestiones de confidencialidad de la información, el banco indicó que el estudio podría continuar solo con el uso de encuestas y no de entrevistas a sus colaboradores.

A pesar de las dificultades mencionadas, las limitaciones permitieron evidenciar el valor de este estudio como contribución a las ciencias de la gestión, aportando de manera relevante a la literatura del tema balance vida-trabajo en las mujeres. En Perú aún no se ha desarrollado tanto el tema, a diferencia de países europeos como España o latinoamericanos 
como Chile y Argentina. En esta línea, existe una tesis sobre la mujer y el balance vidatrabajo en el contexto peruano (Álvarez et al., 2016), la cual trabaja el tema de cómo las mujeres de altos cargos tienden al balance vida-trabajo; sin embargo, dicho estudio tiene un enfoque organizacional -a diferencia de este- que recoge más bien las percepciones de las mismas colaboradoras. En este sentido, este artículo muestra los conocimientos y la importancia para los bancos en el Perú de la mejora de la gestión de su personal femenino, alineándola con sus intereses y sus propias percepciones como colaboradoras como punto de partida. Por tanto, el área de Gestión del Talento puede tomar decisiones sobre las estrategias de atracción y retención de cara a un público que será relevante en los próximos años (jóvenes) y enfrenta situaciones de desventaja ante otros grupos (mujeres).

Por otro lado, este estudio cobra importancia a nivel metodológico por la construcción del instrumento denominado Nimica, en el cual se contextualizan las prácticas de balance vida-trabajo a la realidad peruana y al sector bancario. En este sentido, se desarrolló una metodología para adaptar la literatura proveniente del ámbito internacional a una realidad específica, y se diseñó un instrumento de medición válido y confiable para analizar las percepciones de las prácticas de conciliación en el sector bancario peruano. Esto fue necesario debido a la escasez de literatura latinoamericana y a la falta de un modelo cuantitativo para medir las percepciones de las prácticas de balance vida-trabajo desde la perspectiva de los colaboradores.

El proceso de construcción del instrumento invita a una serie de reflexiones. Este comenzó con la revisión de la literatura de las realidades europeas con el objetivo de adecuarlas al contexto latinoamericano y, posteriormente, al sector bancario peruano. Se partió de una lista de 37 prácticas de conciliación que, tras el análisis de expertos en conciliación, especialistas en recursos humanos y especialistas del sector bancario, se redujo a diez prácticas de conciliación. Es por ello que el resultado del listado de prácticas de balance vida-trabajo para la construcción del instrumento Nimica muestra las diferencias en la gestión de estas prácticas en el contexto europeo y peruano.

Entre las principales diferencias de la implementación de las prácticas del balance vida-trabajo en Perú y otros países, se encontró que dichas prácticas no están institucionalizadas en el país. Asimismo, la ley peruana no las regula, a diferencia de otros países. Por ejemplo, en Europa, el marco legal regula los beneficios que las empresas ofrecen; mientras que en Perú el Estado solo invita a las empresas a incluir este tipo de prácticas. En ese sentido, se tuvo un acercamiento con seis de los principales bancos peruanos y, mediante entrevistas exploratorias, se determinó que cuatro de ellos no tenían prácticas de balance vida-trabajo institucionalizadas y que solo uno orientaba sus prácticas exclusivamente a mujeres, pero estas estaban netamente relacionadas a la maternidad. El otro banco $(A B C)$ es el que ha participado en el presente caso de estudio. Por otro lado, en países como España y Francia la gestión de las prácticas de conciliación se considera una estrategia empresarial, por lo que existe un presupuesto para su diseño, ejecución y control. A diferencia de dichos países, en Perú las empresas realizan prácticas de conciliación de la vida laboral de forma reactiva y las gestionan empíricamente.

Respecto a los hallazgos, se identificaron similitudes y diferencias a partir de los resultados del estudio y lo que por teoría se encontró de las prácticas del balance vidatrabajo y de los determinantes. 
La práctica "actividades recreativas para los hijos» fue una de las menos valoradas. Esto es consistente con lo que sostiene la literatura, enfatizando que las políticas que favorecen la maternidad no han obtenido tanta aceptación como se esperaba en América Latina (Navarro et al., 2012). Sin embargo, es probable que este resultado sea sesgado, pues la mayoría de las mujeres encuestadas no tenían hijos.

Asimismo, se encontró que la práctica «pago de cobertura de EPS» fue considerada como la más importante. Es necesario mencionar que, según entrevistas exploratorias, esta es una práctica común en el sector bancario, por lo que no ofrecerla podría representar una desventaja. De la misma forma, los resultados reconocieron la importancia de las prácticas de flexibilidad para este grupo etario, lo que es consistente con la teoría (Vera, 2017).

Respecto al análisis correlacional, se encontró que, a diferencia de lo que menciona la teoría, para estudiar a la mujer se debe analizar previamente las determinantes de manera conjunta. Es así como se identifican perfiles que afectan la percepción de importancia y existencia respecto a las prácticas del balance vida-trabajo. Al generar perfiles, se resalta la importancia de identificar las particularidades y características de las colaboradoras para la implementación de estrategias y prácticas de balance vida-trabajo.

Se encontró que la determinante "edad» es la más significativa dentro del perfil, pero puede variar según los rangos. Lo expuesto por EY (2017) resalta que existen dos subgrupos entre los jóvenes de 18 a 35 años, cada uno de ellos con sus propias características. Sin embargo, en esta investigación se identificó tres subgrupos, denominados perfiles. De la misma manera, futuras investigaciones sobre este tema en Perú podrían estudiar a mujeres de un mismo grupo de edad, pero insertas en diferentes realidades laborales como empleos de entrada, puestos no ejecutivos y el mercado laboral informal.

Otro resultado en los perfiles fue que la determinante "número de hijos» no es significativa. Según la literatura, ser madre es el rol que demanda más tiempo y dedicación de la mujer fuera del mundo laboral, por lo que es un elemento importante al considerar a la mujer dentro de la esfera del trabajo (Gómez, 2016). No obstante, en este estudio se encontró que se valora menos la práctica directamente relacionada con la maternidad, lo que abre un debate sobre los roles o intereses de la mujer en diferentes países en la actualidad, que no necesariamente están relacionados con ser madre.

Además, se encontró que las determinantes de la mujer, trabajadas de forma conjunta por medio de perfiles, sí afectan la percepción de importancia y existencia respecto a las prácticas de balance vida-trabajo. Es así que se hace evidente la necesidad de tomar en cuenta las particularidades de los colaboradores y sus características propias ante la estrategia e implementación de este tipo de prácticas. Por otro lado, los perfiles identificados también pueden ser utilizados para estudios futuros. Finalmente, en cuanto a las determinantes, estos pueden seguir siendo contemplados en la investigación de mujeres dentro del contexto peruano.

Este estudio abre caminos para futuras exploraciones. En cuanto al instrumento Nimica, los investigadores pueden aplicarlo en otras empresas del sector bancario del Perú o utilizar la metodología para adaptar Nimica a otros sectores o países. También pueden aplicarlo con hombres dentro del mismo rango de edad y realizar un análisis comparativo de ambos sexos en cuanto a sus similitudes o diferencias. Es preciso subrayar que otras determinantes o variables que se alinean con los roles que desempeñan las mujeres 
podrían ser materia de investigación. Asimismo, los datos recopilados con Nimica también pueden ser analizados mediante otras pruebas estadísticas, como los coeficientes beta y beta exponencial, con el fin de profundizar el análisis en términos de dirección y profundidad.

\section{Conclusiones}

Las conclusiones obtenidas del proceso de investigación y de las variables analizadas, las percepciones de existencia y la importancia sobre las prácticas de balance vida-trabajo ejecutadas por el banco ABC por parte de las colaboradoras, serán expuestas en las siguientes líneas.

En primer lugar, a partir del proceso de análisis de las variables se puede comentar que si bien la literatura sostiene que las determinantes influyen en la percepción de las personas respecto a las prácticas del balance vida-trabajo, el presente estudio muestra que estas determinantes no pueden ser estudiadas como variables independientes pues poseen una asociación estadística entre ellas, lo que hace necesaria la creación de perfiles para el análisis correlacional.

En segundo lugar, respecto a la percepción de existencia, se concluye que en dos casos está condicionada por los perfiles; es decir, la percepción de existencia de la práctica puede variar según el perfil al que corresponda. Esta variación puede ser motivo de futuras investigaciones.

En tercer lugar, respecto a la percepción de importancia, se puede decir que esta puede variar según el perfil de la colaboradora o la percepción de existencia de dicha práctica en la empresa. Con la finalidad de enriquecer la investigación, se espera que se aplique este estudio (o la herramienta Nimica) en poblaciones más extensas con el fin de eliminar sesgos relacionados al tamaño reducido de los grupos.

Finalmente, la investigación ha permitido notar la importancia de que las organizaciones conozcan los intereses, características y percepciones de sus colaboradoras, pues resulta relevante para la percepción de ellas respecto a las prácticas de balance vidatrabajo. Para la mujer, las prácticas de balance vida-trabajo representan una oportunidad de lidiar con la esfera laboral; por lo tanto, se sugiere continuar investigando el tema con el fin de que las empresas puedan generar más prácticas a medida de los perfiles que se encuentran dentro de cada organización. En este sentido se agradece a la profesora Paloma Martínez - Hague y a Jorge H. por su apoyo incondicional en el desarrollo de toda la investigación. 


\section{bibliografía}

Álvarez, I., Lozano, M., Morillo, T., \& Ricketts, J.

2016 Balance vida-trabajo de las mujeres ejecutivas que trabajan en empresas privadas en Lima Metropolitana [Tesis de maestría, Centrum Católica]. Recuperado de http://tesis.pucp.edu.pe/repositorio/ handle/123456789/7524

\section{Aequales}

2018 Comunidad Aequales, organizaciones por la equidad de género. Recuperado de https:// aequales.com/comunidad/

\section{Asociación de Bancos del Perú} (Asbanc)

Memoria anual 2015. Recuperado de https://www.asbanc.com.pe/Publicaciones/ Memoria_ASBANC\%202015.pdf

\section{Bendaravičienèb, $\mathbf{R}$.}

Employer brand development measuring organizational attractiveness in higher education institutions [Tesis de doctorado, Vytautas Magnus University]. Recuperado de https://vb.vdu.lt/object/elaba:2182761/

\section{Comisión Económica para} América Latina y el Caribe (CEPAL)

Familias y políticas públicas en América Latina: una historia de desencuentros. Recuperado de https://repositorio. cepal.org/bitstream/handle/11362/2504/ S0700488_es.pdf

\section{Comisión Económica para} América Latina y el Caribe (CEPAL) recuperación. Recuperado de https://www. cepal.org/sites/default/files/document/files/ cuidados_covid_esp.pdf

\section{Chinchilla, N.}

2009 ¿Pintamos algo las mujeres en el mundo empresarial? Recuperado de https://blog. iese.edu/nuriachinchilla/files/2009/02Nerart\% C3\%ADculo5.pdf

Chinchilla, N., \& Jiménez, E.

2013 El papel de la mujer en la nueva economía. Recuperado de https://blog. iese.edu/nuriachinchilla/files/2009/02/ El-papel-de-la-mujer-en-la-nuevaeconom\%C3\%ADa-Catalu\%C3\%B1aecon\% C3\%B3mica-04.04.13.pdf

Chinchilla, N., \& Las Heras, M.

2011 Índice de entornos Familiarmente Responsables (eFR) en el mundo y en Perú [PPT].

Chinchilla, N., Poelmans, S., \& León, $\mathrm{C}$.

2003 Políticas de conciliación trabajo-familia en 150 empresas españolas. Recuperado de https://media.iese.edu/research/pdfs/ Dl-0498.pdf

\section{Clark, S.}

Work/Family Border Theory: A New Theory of Work/Family Balance. Human Relations, July. https://doi. org/10.1177/0018726700536001

\section{Conesa, P., \& Ferrer, P.} Crecimiento profesional y competitividad diferenciada. Una fundamentación antropológica. Recuperado de https:// studylib.es/doc/7261869/versi\%C3\%B3npara-imprimir 


\section{bibliografía}

Del Castillo, E., Fuchs, R., Vera, S., Arizkuren, A., \& Agarwala, T.

2011 Balance trabajo familia: Cultura, nivel de conflicto y voluntad de permanencia en la empresa. Journal of Business, 3(1), 3-14.

\section{El Peruano}

29 de El 52.5\% de la fuerza laboral de los bancos noviem- son mujeres. Recuperado de https://

bre de elperuano.pe/noticia/64300-el-525-de-la-

2017 fuerza-laboral-de-los-bancos-son-mujeres

\section{Ernst\&Young (EY)}

2017

La fuerza laboral de la siguiente generación: ¿un arma secreta o un gran reto? Recuperado de https://www.ey.com/ Publication/vwLUAssets/ey-fuerzalaboralsiguiente-generacion/\$FILE/ey-fuerzalaboral-siguiente-generacion.pdf

\section{Field, A.}

Discovering Statistics Using SPSS 16 ta edición). Londres: SAGE Publications.

\section{Fraj, E.}

Influencia de las características

psicográficas y de conocimiento en

el comportamiento del consumidor

ecológico. Una aplicación empírica [Tesis de doctorado, Universidad de Zaragoza].

Recuperado de https://www.aragon.

es/documents/20127/674325/TESIS_E_

FRAJ_2004.pdf/4962c899-5991-75f6-9002-

6 bf11cccea90

\section{Futuro Labs}

Los Millennials de Lima y el uso del Smartphone. FuturoLabs. Recuperado de http://www.futurolabs.com/los -millennialsde-lima-y-el-uso-del-smartphone/

\section{Gestión}

6 de

No se sabe si un millennials será

agosto un emprendedor o gerente general.

de 2013 Recuperado de https://es.scribd.com/ doc/159503973/Er-Ronald-Estudio-sobrelosmillennials-peruanos-en-diario-Gestion

\section{Gestión}

2017a El 25\% de millennials peruanos asumen puestos de alta jerarquía. Recuperado de https://gestion.pe/tendencias/ millenials-25-peruanos-jovenesasumen-puestos-alta-jerarquia-139751noticia/\#: :text=Seg\%C3\%BAn\%20 un $\% 20$ estudio\%20de\%20Aptitus,y\%20 el\%2018\%25\%20como\%20practicantes.

\section{Gestión}

2017b Día Internacional de la Mujer: 7.4 millones de mujeres conforman la fuerza laboral. 7 de marzo. Recuperado de https://gestion. pe/tendencias/management-empleo/diainternacional-mujer-7-4-millones-mujeresconforman-fuerza-laboral-130204

\section{Great Place to Work (GPTW)}

2018

Great place to work para millennials:

Empresa ABC.

\section{Greenhaus, J., \& Beutel, N.}

1985

Sources of conflict between work and family roles. Academy of Management Review, 10(1), 76-88.

Hernández, R., Fernández, C., \& Baptista, M.

2014 Metodología de la investigación (6ta edición). México D. F.: McGraw Hill Interamericana. 


\section{bibliografía}

\section{INSEAD}

2004

First global research from INSEAD

confirms millennials are misunderstood.

Recuperado de https://www.insead.

edu/news/2014-first-global-research-

from-insead-confirms-millennials-are-

misunderstood

\section{Ipsos}

2018

Perfil del adulto joven del 2018.

Recuperado de https://www.ipsos.com/es-

pe/perfil-del-adulto-joven-peruano-2018

\section{León, $\mathrm{C}$.}

Mujeres y Hombres de Igual a Igual.

Recuperado de http://www.bizkaia.eus/

home2/Archivos/DPTO3/Temas/Pdf/

berdintasuna_2004_5.pdf?hash=a35a66b

b8b7c1ccfe 4b4658e132c2d5

c\&idioma $=\mathrm{CA}$.

\section{Luna-Arocas, R., \& Li-Ping, T.}

The use of cluster analysis to segment clients of a sport center in spain. European Sport Management Quarterly, 5(4), 381413.

Maese, J., Alvarado, A., Valles,

\section{D., \& Báez, Y.}

2016 Coeficiente alfa de Cronbach para medir la fiabilidad de un cuestionario difuso. Cultura Científica y Tecnólogica, 13(59), 146-156.

\section{ManpowerGroup}

2013 El resurgimiento de la escasez de talento: Acciones a tomar para una fuerza laboral sostenible 2013. Recuperado de http://www. upao.edu.pe/bolsadetrabajo/dcto/talento.pdf

\section{ManpowerGroup}

manpower.com.pe/mpintranet/

publicaciones/4466-9358284738328.pdf

\section{Martínez, J., \& Hansen, A.}

2018 alta dirección en las Mipymes de la Ciudad de Tapachula, Chiapas. En $24^{\circ}$ Congreso Nacional sobre Empoderamiento Femenino [Ponencia]. Universidad Autónoma del Estado de Hidalgo. Recuperado de https://uaeh.edu.mx/xiv_congreso_ empoderamiento_fem/memoria.html

Navarro, C., Chinchilla, N., \& Las Heras, $\mathbf{M}$.

Impacto de las políticas y prácticas de conciliación familiar trabajo en organizaciones venezolanas. Revista sobre Relaciones Industriales y Laborales, 48 , 9-26.

Navassi. G.

2003 ¿Cómo gestionar y retener al Talento Humano según su generación? 5 Consultores. Recuperado de http://www.5consultores.com/ gestionarporgeneracion/

\section{Organización Mundial del Trabajo (OIT)}

2018 Perspectivas sociales empleo mundo Recuperado de https://www.ilo.org/ wcmsp5/groups/public/---dgreports/-dcomm/---publ/documents/publication/ wcms_631466.pdf

\section{Otzen, T., \& Manterola, C.}

Técnicas de Muestreo sobre una Población a Estudio. Int. J. Morphol., 35(1), 227-232. Recuperado de https://scielo.conicyt.cl/pdf/ ijmorphol/v35n1/art37.pdf 


\section{bibliografía}

Parada, E., Soto, M., \&

González, D.

2016

El balance vida-trabajo en las

organizaciones de diferentes países en el mundo. EPISTEMUS, (20), 90-94.

\section{Programa de las Naciones}

Unidas para el Desarrollo (PNUD)

2020 El Coronavirus y los retos para el trabajo de las mujeres en América Latina. PNUD América Latina y el Caribe. Recuperado de https://www.latinamerica.undp.org/ content/rblac/es/home/library/crisis_ prevention_and_recovery/el-coronavirus-ylos-retos-para-el-trabajo-de-las-mujeres-ename.html

\section{Ponce, M., \& Pasco, M.}

2015 Guía de Investigación-Gestión. Lima:

Fondo Editorial PUCP. Recuperado de https://www.researchgate.net/ publication/326478701_GUIA_DE_ INVESTIGACION_EN_GESTION

\section{Pomar, S., \& Martínez, G.}

Resignificación identitaria, trabajo y familia: una disyuntiva para la mujer. Administración y organizaciones, 9(18), 95-109.

\section{Ramírez, K.}

La implementación de la responsabilidad familiar corporativa mejora el bienestar de los trabajadores: Caso Caja Rural de Ahorro y Crédito Raíz [Tesis de posgrado, Universidad Peruana de Ciencias Aplicadas]. Recuperado de http://hdl. handle.net/10757/623568

\section{Red Concilia}

2007

Guía de prácticas de Conciliación de la Vida Personal, Familiar y Laboral en las Empresas. Recuperado de https:// www.unirioja.es/igualdad/archivos/Guia_ Conciliacion_Buenas_Practicas.pdf

\section{Ministerio de Trabajo y Promoción del Empleo del Perú (MTPE)}

RES Na. 048-2014-TR. Guía de buenas prácticas en materia de conciliación del trabajo y la vida familiar y personal. Recuperado de https://es.scribd.com/ document/373314376/Guia-de-buenaspracticas-en-materia-de-conciliacion-deltrabajo-y-la-vida-familiar-y-personal

\section{Salgado, M.}

Balance vida-trabajo de las mujeres gerentes que trabajan en empresas privadas del área metropolitana de Caracas [Tesis de licenciatura, Universidad Católica Andrés Bello]. Recuperado de http:// biblioteca2.ucab.edu.ve/anexos/biblioteca/ marc/texto/AAS3043.pdf

\section{Superintendencia de Banca y Seguros (SBS)}

Programa de finanzas en el cole: Programa de asesoría a docentes sobre el rol y funcionamiento del sistema financiero de seguros, AFP y Unidades de inteligencia financiera - Guía del docente. Lima: Superintendencia de Banca y Seguros, Ministerio de Educación. Recuperado de http://www.sbs.gob.pe/portals/3/ educacion-financierapdf/Guia \%20del\%20 docente\%202017.pdf 


\section{bibliografía}

Vera, I.

2017 Balance vida - trabajo. Lima: Departamento

Económico de Ciencias de la Gestión

de la Pontificia Universidad Católica

del Perú. Recuperado de http://

repositorio.pucp.edu.pe/index/bitstream/

handle/123456789/111951/Balance_Vida_

Trabajo.pdf? sequence=1\&isAllowed=y

\section{Zúñiga, G.}

2002 Estudio de los determinantes de la

participación femenina en el mercado

laboral a través de un análisis multivariado

factorial. Revista temas de coyuntura,

(46), 85-112. Recuperado de http://

revistasenlinea.saber.ucab.edu.ve/temas/

index.php/temasdecoyuntura/article/

view/1685.

Fecha de recepción: 13 de julio de 2020

Fecha de aprobación: 26 de noviembre de 2020 